International Journal of Pure and Applied Mathematics

Volume 92 No. 5 2014, 745-755

ISSN: 1311-8080 (printed version); ISSN: 1314-3395 (on-line version)

url: http://www.ijpam.eu

doi: http://dx.doi.org/10.12732/ijpam.v92i5.9

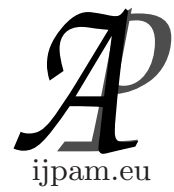

\title{
NON-LINEAR ENERGY HARVESTING SYSTEM EFFICIENCY COMPARISON FROM PERIODIC TO NON-IDEAL EXCITATION
}

\author{
Douglas da Costa Ferreira ${ }^{1 \S}$, Fábio Roberto Chavarette ${ }^{2}$, \\ Nelson José Peruzzi ${ }^{3}$ \\ ${ }^{1}$ UNESP - Univ. Estadual Paulista \\ Mechanical Engineering PhD Program \\ Brasil Avenue, 56, 15385-000, Ilha Solteira, SP, BRAZIL \\ ${ }^{2}$ UNESP - Univ. Estadual Paulista \\ Mathematics Department \\ Brasil Avenue, 56, 15385-000, Ilha Solteira, SP, Brazil \\ ${ }^{3}$ UNESP - Univ. Estadual Paulista \\ Mathematics Department
}

Prof. Paulo D. Castellane street, 14884-900, Jaboticabal, SP, BRAZIL

\begin{abstract}
Dynamics of a non-linear energy harvesting is affected by excitation source and to explore efficiency it is necessary to understand which region offers more energy and the influence of a forced periodic vibration related to ambient non-ideal and power source. A bistable Duffing oscillator coupled to piezoelectric layers and electric load configuring a non-linear energy harvesting is excited by periodic resonant vibration and the system behaviour and energy availability is compared to non-ideal excitation. The chaos via Lyapunov exponent were determined and Runge-Kutta forth order was applied to determine displacement, velocity, phase portrait and output voltage. As main conclusion it was possible to identify a more efficient coupling of cantilever configuration to non-ideal power source than resonant periodic excitation. Considering the kinetic energy available for the coupled system to be converted into electricity it was possible to verify an increase around then times of energy offered to the harvesting system from non-ideal vibration compared to periodic resonant
\end{abstract}

\footnotetext{
Received: March 13, 2014

(c) 2014 Academic Publications, Ltd. url: www.acadpubl.eu

${ }^{\S}$ Correspondence author
} 
design.

AMS Subject Classification: $74 \mathrm{H} 65$

Key Words: non-linear energy harvesting, periodic excitation, non-ideal excitation, efficiency, dynamics

\section{Introduction}

The growth for electricity power consumption requires better ways of supplying and one of the promising technologies are Energy Harvesting that is even a sustainable solution. Among several harvesters proposed three main groups are highlighted: thermal gradients, solar radiation and vibration [1]. Considering vibration case the main subject is transfer kinetic energy from some source to a converter into electricity. This conversion can be accomplished via many solutions and piezoelectric materials are the most promising [2].

Piezoelectric material converts motion to electricity considering his direct effect and several studies regards piezoelectric layers attached to a object vibrating and the piezoelectric material is coupled to a electrical load [3]. It configures a simple solution however the system efficiency is considerable low regards vibrational interaction to the power source as the main cause [4]. To enhance harvesting efficiency it is necessary offers more kinetic energy from excitation source to piezoelectric layers to be converted into electricity and to accomplished this objective some dynamical solution is necessary. In this direction first studies concentrates in matching natural frequency to excitation source for purpose of resonance from vibration source to excited object [2]. This dynamical solution reached their expected result nevertheless mostly ambient vibration are in wide band and their frequency is random which hampers a design solution for resonance [3], [4],[5] and [6].

To trespass the resonance matching problem several non-linear solution was proposed [5] ant [6] and has spotlight the bistable cantilever proposed by [6] that regards to a cantilever subject to two magnets creating a tuning design. The bistable configuration results in non-linear behaviour from harvester configuration. It was studied the dynamics from non-linear energy harvesting excited by periodic excitation. For maximization the kinetic absorption from excitation source to beam coupled to piezoelectric layers a resonant design were the object of study by [6].

To explore energy availability it is proposed in this research a non-ideal vibration as excitation configuring a non-linear harvester coupled to a nonideal power source. For limited power the feedback vibration influences the 


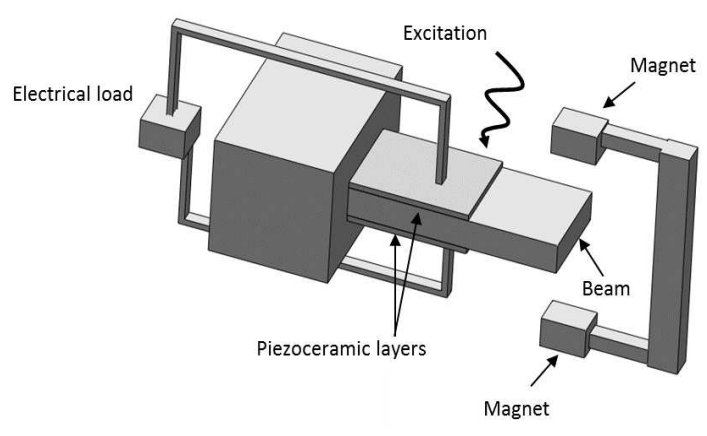

Figure 1: Harvester with periodic excitation

external source which characterizes a non-ideal system [7]. Thus the model of movement sums a feedback term increasing the problem degrees of freedom [8]. According [9] and [10] when vibration source is near natural frequency for nonlinear vibrational systems occurs a jump phenomenon known as Sommerfeld effect [11] making not possible to reach the resonance as maximum vibration response.

Considering the paper organization: Section 2 presents the modelling of non-linear harvester subject to a periodic excitation, Section 3 presents the modelling of non-linear harvester excited by a non-ideal power source and Section 4 presents de efficiency comparison of two excitation sources and acknowledgements.

\section{Non-Linear Harvester Excited by Periodic Vibration}

A cantilever covered with two layers of piezoelectric film is subject to a periodic excitation $f \cos \Omega t$. To set up a bistable behaviour to magnets impose a nonliner behaviour, as shown in Fig. 1. This harvester proposed by [6] is a Duffing oscillator for increasing the frequency bandwidth and take advantage of full range broadband. The periodic vibration as excitation source matching the natural frequency from coupled system as a resonant design.

Considering periodic excitation as $f \cos \Omega t$ the equation of motion from this harvester configuration and electrical coupling is given by equation (1) according $[6]$.

$$
\ddot{x}+2 \zeta \dot{x}-\frac{1}{2} x\left(1-x^{2}\right)-\chi \nu=f \cos \Omega t
$$




$$
\dot{\nu}+\Lambda \nu+\kappa \dot{x}=0
$$

Where $\zeta$ is damping, $\chi$ is piezoelectric mechanical coupling coefficient, $\nu$ is resistance voltage, $\lambda$ is reciprocal of time constant and $\kappa$ is piezoelectric electric coupling coefficient. The state variable $x$ refers to beam position.

Defining $x_{1}=\mathrm{x}$ and $x_{3}=\nu$ than the space-state of equations (1) is given by equation (2).

$$
\begin{array}{r}
\dot{x_{1}}=x_{2} \\
\dot{x_{2}}=\frac{1}{2} x_{1}\left(1-x_{1}^{2}\right)-2 \zeta x_{2}+\chi x_{3}+f \cos \Omega t \\
\dot{x_{3}}=-\kappa x_{2}-\Lambda x_{3}
\end{array}
$$

\subsection{Dynamical Analysis for Periodic Excitation}

This section presents the dynamic evaluation for bistable harvester subject to periodic excitation. This configuration is largely known in literature and completely discussed in [6]. To summary the dynamic study is presented the stability, displacement rate, phase portrait and output voltage. For this study it is used parameters for resonance design given in table 1 according [6].

\begin{tabular}{ccccccc}
\hline Parameters & $\zeta$ & $\chi$ & $\Lambda$ & $\kappa$ & $f$ & $\Omega$ \\
\hline Value & 0.01 & 0.05 & 0.05 & 0.5 & 0.083 & 0.8 \\
\hline
\end{tabular}

Table 1: Parameters for resonance [6]

The Lyapunov Exponents calculated via Wolf Method shows a chaotic behaviour of bistable configuration shown in Fig. 2. This configuration varies his behaviour when frequency excitation is changed and the energy peak is around chaotic region [12]. The behaviour also depends of excitation amplitude and coupling damping [13].

\begin{tabular}{cc}
\hline$\lambda_{1}$ & 0.08978 \\
\hline$\lambda_{2}$ & -0.069944 \\
\hline$\lambda_{3}$ & -0.089836 \\
\hline
\end{tabular}

Table 2: Lyapunov exponents for periodic excitation - Values 


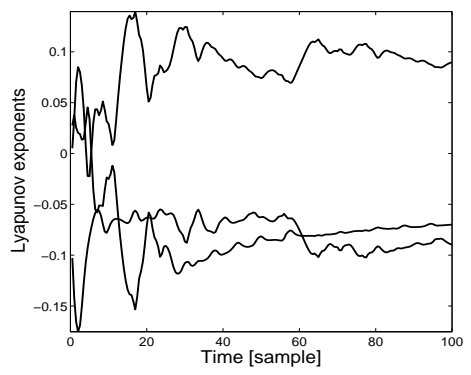

Figure 2: Lyapunov Exponents for periodic excitation - Time sample

(a)

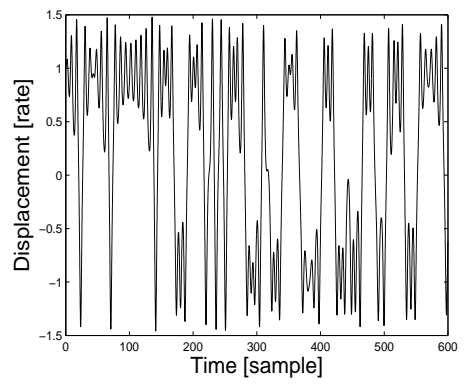

(c)

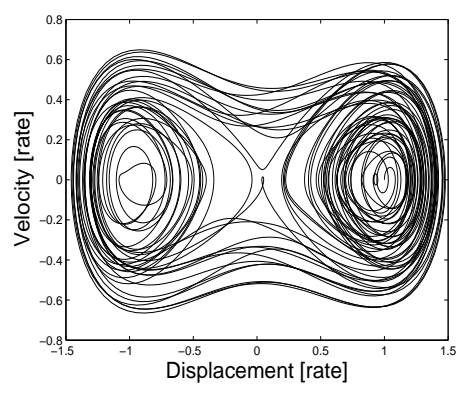

(b)

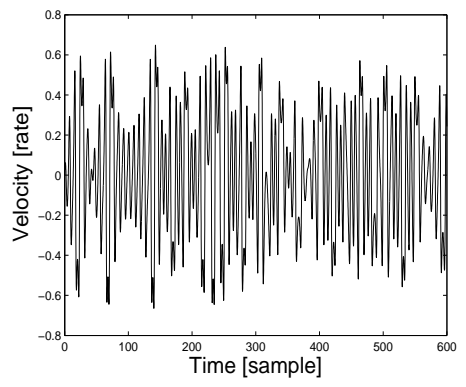

(d)

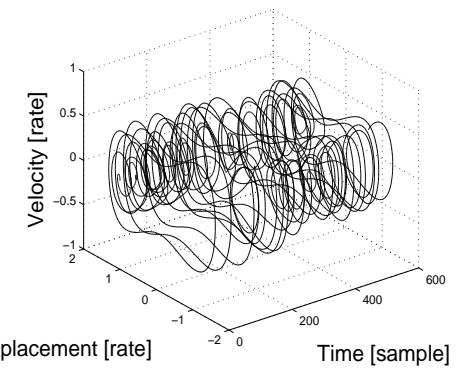

Figure 3: Dynamical behavior for periodic excitation (a) displacement rate, (b) velocity rate, (c) phase portrait, (d) time sample phase portrait

Performing analysis via Runge-Kutta fourth order method for solving ordinary differential equations it is possible to visualize the total energy from the system. Fig. 3 exhibit results from 0 to 600 samples in 0.1 steps resulting in 6,000 samples. 


\section{Non-Linear Harvester Excited by Non-Ideal Power Source}

The equation of motion and electrical coupling for non-ideal excitation $q(\dot{z} \cos z+$ $\ddot{z} \sin z$ ) is given by equation (3) according to [7] and [15].

$$
\begin{array}{r}
\ddot{x}+2 \zeta \dot{x}-\frac{1}{2} x\left(1-x^{2}\right)-\chi \nu=q(\dot{z} \cos z+\ddot{z} \sin z) \\
\ddot{z}+b \dot{z}=r \ddot{x} \sin z+a \\
\dot{\nu}+\Lambda \nu+\kappa \dot{x}=0
\end{array}
$$

where $q$ is non-ideal excitation and $b, r$ and $a$ refers to non-ideal behaviour parameters. The state variable $z$ refers to vibration source position. Isolating $\ddot{x}$ and $\ddot{z}$ the equations for coupling are described by equation (4).

$$
\begin{array}{r}
\ddot{x}=\frac{\frac{1}{2} x\left(1-x^{2}\right)-2 \zeta \dot{x}+\chi \nu+q \dot{z}^{2} \cos \dot{z}+q \sin \dot{z}(a-b \dot{z})}{1-q r \sin ^{2}(z)} \\
\ddot{z}=\frac{r \sin \dot{z}\left(\frac{1}{2} x\left(1-x^{2}\right)-2 \zeta \dot{x}+\chi \nu+q \dot{z}^{2} \cos \dot{z}\right)+a-b \dot{z}}{1-q r \sin ^{2}(z)} \\
\dot{\nu}=-\kappa \dot{x}-\Lambda \nu
\end{array}
$$

Adopting $x_{1}=\mathrm{x}$ and $x_{3}=z$ and $x_{5}=\nu$ than the space-state of equation (4) is given by equation (5).

$$
\begin{array}{r}
\dot{x_{2}}=\frac{\frac{1}{2} x_{1}\left(1-x_{1}^{2}\right)-2 \zeta x_{2}+\chi x_{5}+q x_{4}^{2} \cos x_{3}+q \sin x_{3}\left(a-b x_{4}\right)}{1-q r \sin ^{2}\left(x_{3}\right)} \\
\dot{x_{3}=x_{4}} \\
\dot{x_{4}}=\frac{r \sin x_{3}\left(\frac{1}{2} x_{1}\left(1-x_{1}^{2}\right)-2 \zeta x_{2}+\chi x_{5}+q x_{4}^{2} \cos x_{3}\right)+a-b x_{4}}{1-q r \sin ^{2}\left(x_{3}\right) \quad} \\
\dot{x_{5}}=-\kappa x_{2}-\Lambda x_{5}
\end{array}
$$

\subsection{Dynamical Analysis for Non-Ideal Excitation}

Designs based in resonant solution takes in consideration a periodic and known vibration source however most ambient vibration is random and unknown [14] which demands a solution for non-linear harvesters and considering a nonperiodic excitation. Studies accomplished by [6] compared performance of linear and non-linear harvester for periodic excitation. In more recent studies 


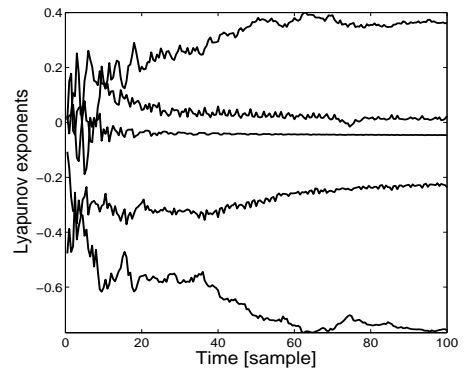

Figure 4: Lyapunov exponents for non-ideal excitation - Time sample

[14] utilizes random excitation to analyse the potential of proposed non-linear harvester. The present research propose a non-ideal power source as system excitation for non-linear harvester. The non-ideal excitation source was modelled by [7] and their behaviour for piezoelectric coupling in linear case is discussed by [15]. For this non-ideal power source coupled to non-linear harvester system the proposed parameter are given in table (3).

\begin{tabular}{ccccc}
\hline Parameters & $a$ & $b$ & $q$ & $r$ \\
\hline Value & 2.3 & 0.5 & 0.5 & 0.5 \\
\hline
\end{tabular}

Table 3: Parameters for non-ideal excitation [6]

Lyapunov Exponents present a chaotic behaviour as shown in Fig 6. According [16] a small increase of energy can convert periodic vibration into chaos. This system have non-negative exponents as shown in table 5 .

\begin{tabular}{cc}
\hline$\lambda_{1}$ & 0.362719 \\
\hline$\lambda_{2}$ & 0.023508 \\
\hline$\lambda_{3}$ & -0.045574 \\
\hline$\lambda_{4}$ & -0.235956 \\
\hline$\lambda_{5}$ & -0.758101 \\
\hline
\end{tabular}

Table 4: Lyapunov exponents for non-ideal excitation - Values

Applying Runge-Kutta forth order for the non-linear harvester excited to non-ideal power source the system presents results as shown in Fig 5 . 
(a)

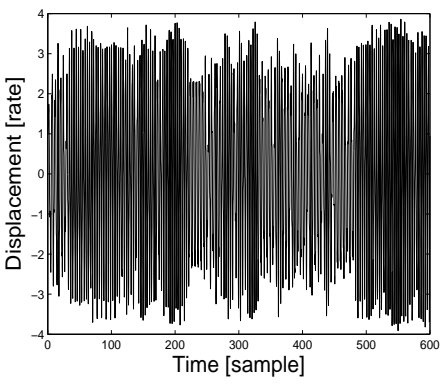

(c)

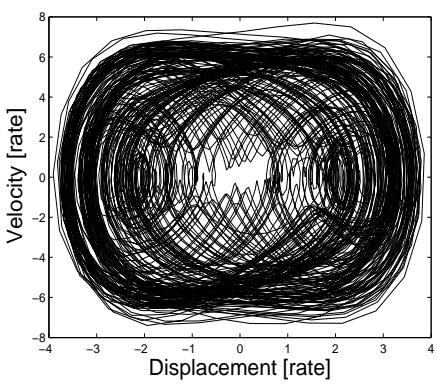

(b)

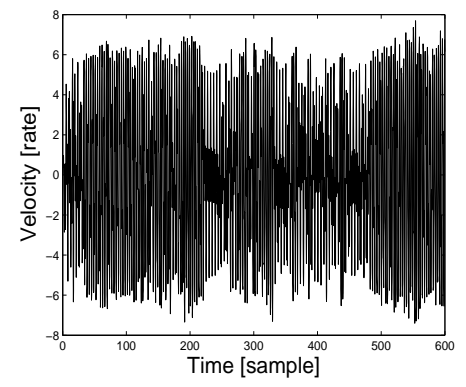

(d) Displacement [rate]

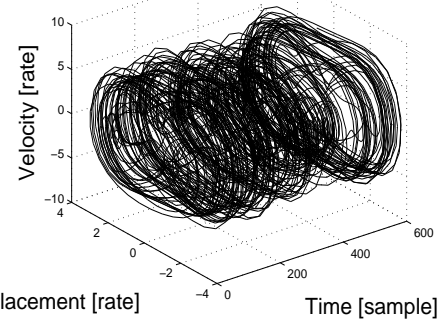

Figure 5: Dynamical behavior for non-ideal excitation (a) displacement rate, (b) velocity rate, (c) phase portrait, (d) time sample phase portrait

\section{Efficiency Comparison}

Piezoelectric direct effect converts kinetic energy to electricity and taking in consideration displacement and velocity as main measure of kinetic energy it is possible to compare efficiency from periodic excitation to non-ideal excitation measuring displacement and velocity rate trough phase portrait graphic which represent total kinetic energy offered to the system as shown in Fig. 6. It was considered 500 samples from 1,500 to 2,000 to exclude transient behaviour.

There was an increase of around then times energy offered to the harvesting system from non-ideal excitation compared to periodic resonant design. This result encourages development of controller systems to set-up harvesters to nonideal behaviour during interaction to a specific power source. 


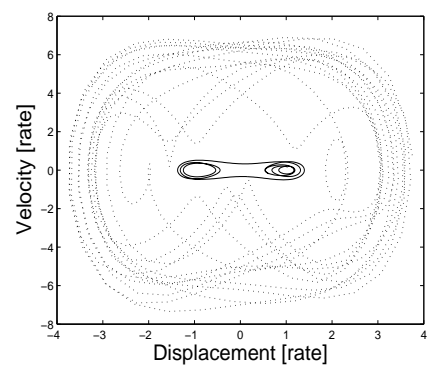

Figure 6: Phase Portrait Comparison - periodic excitation (solid line) and non-ideal excitation (dotted line) - Samples from 1500 to 2000

\section{Acknowledgments}

The authors would like to thank to CNPq (Proc. n. 301769/2012-5) and Federal University of Mato Grosso (UFMT) for funding this research.

\section{References}

[1] S. Roundy, P. K. Wright, J.Rabaey, A study of low level vibrations as a power source for wireless sensor nodes, Computer Communications, 26, (2003), 11311144.

[2] A. Harb, Energy harvesting: State-of-the-art, Renewable Energy, 36, (2011), 2641-2654, doi:10.1016/j.renene.2010.06.014.

[3] F. Goldschmidtboeing, P. Woias, Characterization of different beam shapes for piezoelectric energy harvesting, Journal of Micromechanics and Microengineering, 18, (2008), 104013, doi:10.1088/0960-1317/18/10/104013.

[4] H. A. Sodano, D. J. Inman, Comparison of Piezoelectric Energy Harvesting Devices for Recharging Batteries, Journal of Intelligent Material Systems and Structures, 16(10), (2005), 799-807.

[5] R. L. Harne, K. W. Wang, A review of the recent research on vibration energy harvesting via bistable systems, Smart Materials and Structures, 22, (2013), doi:10.1088/0964-1726/22/2/023001.

[6] A. Erturk, D. J. Inman, Broadband piezoelectric power generation on highenergy orbits of the bistable duffing oscillator with electromechanical cou- 
pling, Journal of Sound and Vibration, 330, No. 10, (2011), 2339-2353, doi: $10.1016 /$ j.jsv.2010.11.018.

[7] F. R. Chavarette, Control Design applied to a non-ideal structural system with behavior chaotic, International Journal of Pure and Applied Mathematics, 86, No. 03, (2013), 487-500, doi: 10.12732/ijpam.v86i3.3.

[8] J. M. Balthazar, D. T. Mook, H. I. Weber, R. M. L. R. F. Brasil, A. Fenili, D. Belato, J. L. P. Felix, An Overview on Non-Ideal Vibrations, Meccanica, 38, (2003), 613621.

[9] I. Iliuk, J. M. Balthazar, A. M. Tusset, J. L. P. Felix, B. R. Pontes Jr., On Non-ideal and Chaotic Energy Harvester Behavior, Differential Equation Dynamical System, 21, (2013), 93104, doi: 10.1007/s12591-012-0127-5.

[10] S. El-Serafi, M. Eissa, T.H. El-Ghareeb, 1:4 Internal Resonance Active Absorber for Non-Linear Vibrating System. International Journal of Pure and Applied Mathematics, 28, No. 1, (2006), 71-84.

[11] M. Tsuchida, K. L. Guilherme, J. M. Balthazar, On chaotic vibrations of a non-ideal system with two degrees of freedom- 1-2 resonance and Sommerfeld effect, Journal of Sound and Vibration, 282, (2005), 12011207, doi: $10.1016 /$ j.jsv.2004.04.025.

[12] L. V. Blarigan, P. Danzl, J. Moehlis, A broadband vibrational energy harvester, Applied Physics Letters, 100, (2012), 253904, doi: $10.1063 / 1.4729875$.

[13] L. V. Blarigan, P. Danzl, J. Moehlis, Exploiting Nonlinearity to Provide Broadband Energy Harvesting, Proceedings of the ASME 2009 Dynamic Systems and Control Conference, 2542, (2009), 12-14.

[14] G. Litak, M. I. Friswell, S. Adhikari, Magnetopiezoelastic energy harvesting driven by random excitations, Applied Physics Letters, 96, (2010), 214103, doi: $10.1063 / 1.3436553$.

[15] I. Iliuk, J. M. Balthazar, A. M. Tusset, J. L. P. Felix, B. R. Pontes Jr, Nonlinear dynamics and control strategies - On an energy harvester vibrating system with a linear form to non-ideal motor torque, MATEC Web of Conferences, 1, (2012), 08003, doi: 10.1051/matecconf/20120108003. 
[16] L. V. Blarigan, P. Danzl, J. Moehlis, Symbolic Dynamics in Chaotic Wave Vibration, International Journal of Pure and Applied Mathematics, 16, No. 2, (2004), 265-280. 
